University of Nebraska - Lincoln

DigitalCommons@University of Nebraska - Lincoln

Faculty Papers and Publications in Animal

Science

Animal Science Department

$12-22-2013$

\title{
Beef cattle body temperature during climatic stress: a genome- wide association study
}

Jeremy T. Howard

University of Nebraska-Lincoln

Stephen D. Kachman

University of Nebraska-Lincoln, steve.kachman@unl.edu

Warren M. Snelling

U.S. Meat Animal Research Center, USDA, warren.snelling@ars.usda.gov

E. John Pollak

U.S. Meat Animal Research Center, USDA, jpollak2@unl.edu

Daniel C. Ciobanu

University of Nebraska-Lincoln, dciobanu2@unl.edu

See next page for additional authors

Follow this and additional works at: https://digitalcommons.unl.edu/animalscifacpub

Part of the Genetics and Genomics Commons, and the Meat Science Commons

Howard, Jeremy T.; Kachman, Stephen D.; Snelling, Warren M.; Pollak, E. John; Ciobanu, Daniel C.; Kuehn, Larry A.; and Spangler, Matthew L., "Beef cattle body temperature during climatic stress: a genome-wide association study" (2013). Faculty Papers and Publications in Animal Science. 863.

https://digitalcommons.unl.edu/animalscifacpub/863

This Article is brought to you for free and open access by the Animal Science Department at DigitalCommons@University of Nebraska - Lincoln. It has been accepted for inclusion in Faculty Papers and Publications in Animal Science by an authorized administrator of DigitalCommons@University of Nebraska Lincoln. 


\section{Authors}

Jeremy T. Howard, Stephen D. Kachman, Warren M. Snelling, E. John Pollak, Daniel C. Ciobanu, Larry A. Kuehn, and Matthew L. Spangler 


\title{
Beef cattle body temperature during climatic stress: a genome-wide association study
}

\author{
Jeremy T. Howard • Stephen D. Kachman • \\ Warren M. Snelling • E. John Pollak • Daniel C. Ciobanu • \\ Larry A. Kuehn • Matthew L. Spangler
}

Received: 7 August 2013 /Revised: 26 November 2013 / Accepted: 27 November 2013 /Published online: 22 December 2013

(C) ISB 2013

\begin{abstract}
Cattle are reared in diverse environments and collecting phenotypic body temperature (BT) measurements to characterize BT variation across diverse environments is difficult and expensive. To better understand the genetic basis of BT regulation, a genome-wide association study was conducted utilizing crossbred steers and heifers totaling 239 animals of unknown pedigree and breed fraction. During predicted extreme heat and cold stress events, hourly tympanic and vaginal BT devices were placed in steers and heifers, respectively. Individuals were genotyped with the BovineSNP50K_v2 assay and data analyzed using Bayesian models for area under the curve (AUC), a measure of BT over time, using hourly BT observations summed across 5-days (AUC summer 5-day (AUCS5D) and AUC winter 5-day (AUCW5D)). Posterior heritability estimates were moderate to high and were estimated to be 0.68 and 0.21 for AUCS5D and AUCW5D, respectively. Moderately positive correlations between direct genomic values for AUCS5D and AUCW5D $(0.40)$ were found, although a small percentage of the top $5 \% 1-\mathrm{Mb}$ windows were in common. Different sets of genes were associated with BT during winter and summer,
\end{abstract}

USDA is an equal opportunity employer. Mention of trade names or commercial products in this publication is solely for the purpose of providing specific information and does not imply recommendation or endorsement by the U.S. Department of Agriculture.

J. T. Howard · D. C. Ciobanu · M. L. Spangler $(\bowtie)$

A218h Animal Science Department, University of Nebraska,

Lincoln, NE 68583-0908, USA

e-mail: mspangler2@unl.edu

S. D. Kachman

Department of Statistics, University of Nebraska, Lincoln, NE 68583, USA

W. M. Snelling $\cdot$ E. J. Pollak $\cdot$ L. A. Kuehn Agricultural Research Service, U.S. Meat Animal Research Center, USDA, Clay Center, NE 68933, USA thus simultaneous selection for animals tolerant to both heat and cold appears possible.

Keywords Beef cattle $\cdot$ Body temperature $\cdot$ Genome-wide association study

\section{Introduction}

Cattle are reared in environments that differ greatly in temperature, humidity, and wind speed, which have forced cattle to be regionally adapted, thus creating sensitivity to environments that differ greatly from the adapted environment. This potentially decreases production efficiency in foreign environments and affects the global use of certain genotypes/germplasm (Hahn 1999; Young 1983) which has been seen in the exchange of regionally adapted Line 1 Hereford from Miles City, MT, USA and Hereford cattle originating from Brooksville, FL, USA (MacNeil 2009). The inability to regulate body temperature (BT) results in energy reserves being diverted to thermoregulatory processes such as decreased or increased heat production during heat stress and cold stress, respectively, thereby reducing energy reserves for growth, lactation or pregnancy (Hahn, 1999). Consequently, developing cattle with a genomic profile that is conducive to performing in diverse environments (i.e., low variation in BT across environments) while maintaining a high level of productivity and possessing superior carcass attributes is advantageous (Scharf et al. 2010).

One possible way to increase environmental tolerance is to characterize animals within a population based on their inherent differences for BT regulation across environments using continuous internal BT measurements. A simulated selection scheme by Nardone and Valentini (2000) compared selection for heat tolerance within a high-milking breed and milk 
production within a highly adapted breed. The authors found that selection for heat tolerance within the high-milking breed was more efficient due to the adapted breed needing several generations (30 plus) to reach comparable levels of milk production. Animal variation has been shown to exist for BT regulation of beef cattle during periods of extreme external temperature, with heritability estimates ranging from 0.11 to 0.44 (Burrow 2001; Da Silva 1973; Turner 1982, 1984). Internal BT is difficult and expensive to measure in a production setting. Thus, identifying and using genetic variants that impact BT regulation for selection and management purposes is highly applicable. Our objective was to conduct a genome-wide association study (GWAS) to discover the genetic basis of $\mathrm{BT}$ regulation during periods of heat and cold stress using continuous BT measurements and to better understand the genetic relationship between these stressors.

\section{Materials and methods}

\section{Experimental design}

This project was approved by the University of NebraskaLincoln Institutional Animal Care and Use Committee. Crossbred calf-fed steers and yearling heifers of unknown pedigree and breed fractions $(n=239)$ with varying percentages of Angus, Simmental, and Piedmontese were fed in four groups over a 2-year period, where a group was defined by sex and year such that there was only one group being evaluated at a time (Howard et al. 2013). Individual animal BT were recorded per Howard et al. (2013). In brief, during anticipated times of heat and cold extremes based on future weather predictions, hourly BT recording devices (resolution of $0.0625^{\circ} \mathrm{C}$ ) were placed for a minimum of 5 days inside the ear canal (tympanic) for steers or intravaginally using a modified blank (i.e., did not contain hormones) controlled internal drug release (CIDR) for heifers. Periods of minimal stress or external temperatures in which the animals would be in their thermoneutral zone was not measured; therefore, an animalspecific average BT could not be estimated. Two types of BT recording devices were used due to increased likelihood of ear infections and the amount of time the device can be placed in the ear (around 7 days) in comparison to devices placed intravaginally, which possess no major health concerns and can be in use for around 21 days. A subset of heifers $(n=8)$ had both tympanic and vaginal BT recorded during a heat stress event and a correlation of 0.98 was estimated between the two. Tympanic temperature averaged $0.163{ }^{\circ} \mathrm{C}$ greater than vaginal temperature; therefore, to account for this difference, steer tympanic BT was subtracted by $0.163{ }^{\circ} \mathrm{C}$. In a study by Bergen and Kennedy (2000), the authors found a phenotypic correlation of $0.77(P<0.05)$ between vaginal and tympanic temperature in nine crossbred heifer calves across multiple recording periods with a minimum of 7 days between recording, whereas in the current study the correlation was derived from a single recording period. During heat stress events, there were 110 and 115 tympanic and vaginal temperature measurements, respectively. During cold stress events, there were 111 and 109 tympanic and vaginal temperature measurements, respectively.

Ambient temperature ( $\mathrm{Ta}$, in degree Celsius), relative humidity (RH, in percent), wind speed (WS, in kilometer per hour), and solar radiation (SR, in kilocalorie per square meter) were taken hourly at a nearby automated weather station as described by Howard et al. (2013). These parameters were used to compute a Comprehensive Climate Index (CCI) that was effective for winter and summer conditions (Mader et al. 2010). The average ( \pm SD) CCI (in degree Celsius) was 24.40 $( \pm 4.64)$ and $-1.80( \pm 11.71)$ for summer and winter stress conditions, respectively. Area under the curve (AUC), a measure of BT over time, was approximated with the Trapezoid rule using hourly BT observations summed across 5 days $(n=$ 120 per animal) during summer (AUCS5D) and winter (AUCW5D) conditions. A high AUC value for a heat stress event or a low AUC value for a cold stress event indicates poor BT regulation. The average $( \pm \mathrm{SD})$ AUC (in degree Celsius $\times$ hour) for AUCS5D and AUCW5D was 4,627.1 $( \pm 34.37)$ and 4,570.9 $( \pm 49.44)$, respectively.

An ear notch (EN) sample was collected from each animal and used for DNA extraction. DNA was isolated from 10 to $25 \mathrm{mg}$ of tissue from each animal using the DNeasy blood and tissue kit (Qiagen). The quantity and quality of the DNA sample was assessed by NanoDrop Spectrophotometer (Thermo Scientific) and agarose gel electrophoresis. Genotyping of the DNA samples was performed using Ilumina BovineSNP50K_v2 Bead-Chip (Illumina Inc., San Diego, CA, USA). All samples used had a call rate above $97.5 \%$. Illumina data analysis software was used to assign quality scores (GenCall) for each genotype. If genotypes were missing or a GenCall score was below 0.20 (Illumina, Inc 2010; Edriss et al. 2012), genotypes were replaced with the mean allele frequency across all animals. Exclusion of markers based on minor allelic frequency and deviations from Hardy-Weinberg proportions were shown by Edriss et al. (2012) to have little impact on genetic prediction and therefore all single-nucleotide polymorphism (SNP) were utilized for analysis. Animals were removed from the analysis for summer $(n=14)$ and winter $(n=13)$ stress events, due to missing hourly BT observations. Additional steer observations $(n=5)$ were removed from the analysis for winter stress events due to BT observations not following a cyclical pattern similar to other animals in the group, likely due to data logger malfunctions. The final number of animals was 225 and 220 for summer and winter stress events, respectively. 


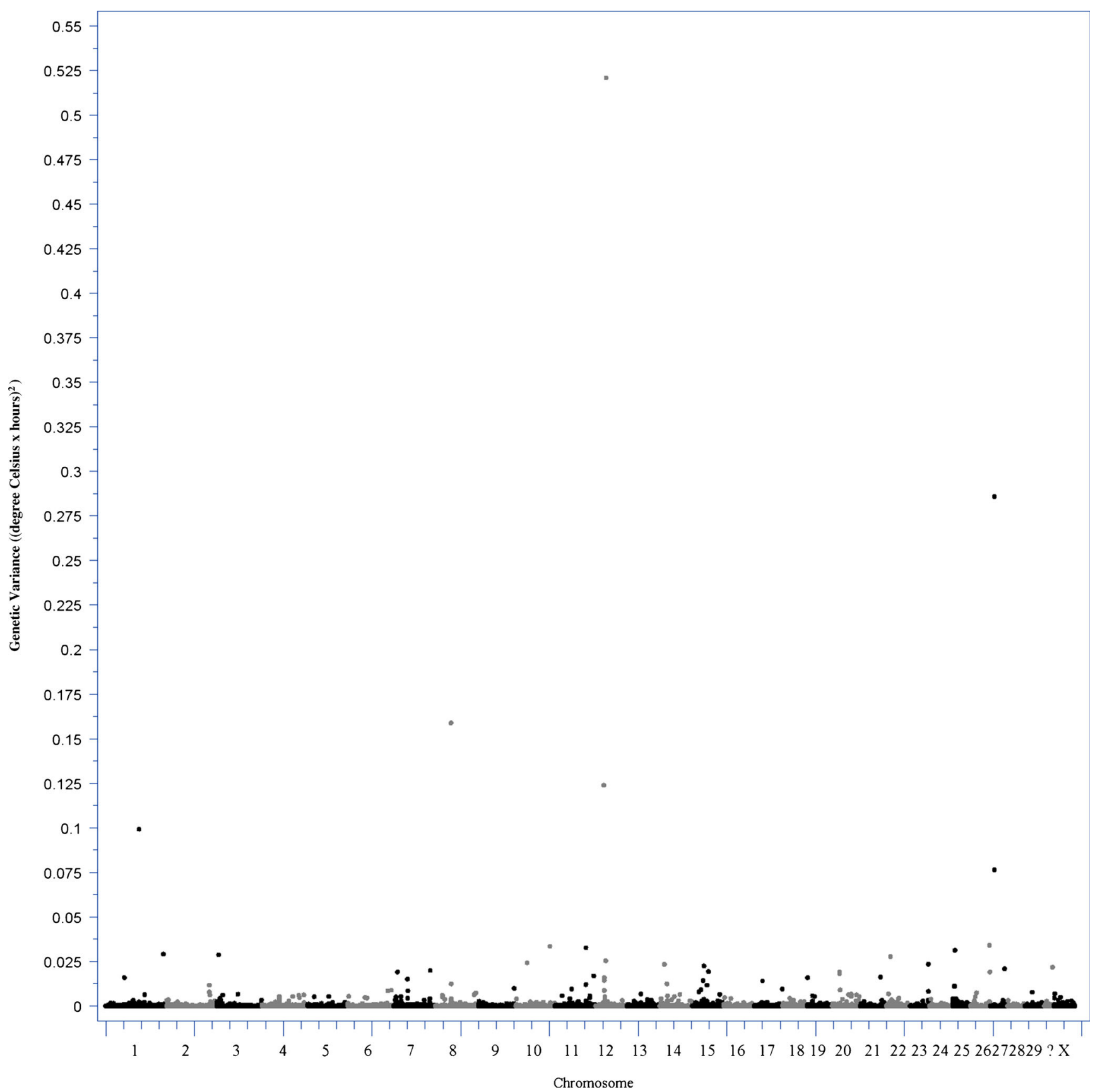

Fig. 1 Genome-wide association analysis between 54,609 SNP and area under the curve across 5 day $(120 \mathrm{~h})$ during summer conditions (AUCS5D). The $Y$-axis represents the contribution of a marker to the
SNP variance ((in degree Celsius $\times$ hours $\left.)^{2}\right)$. On the $X$-axis, alternate gray and black colors represent different chromosomes (chromosome refers BTA1 to BTA29, followed by SNP with unknown location (?)

\section{Statistical analysis}

A GWAS using 5-day AUC for summer and winter stress events was undertaken to estimate the proportion of phenotypic variation in AUC for both stress periods that was explained by the markers. Estimates of marker effects and variances were obtained by fitting all markers simultaneously using Bayesian methods via GenSel (version 0.9.2.045; Fernando and Garrick 2009). The mixed model to determine marker effects and variances can be represented as: $y=X \beta+\sum_{k=1}^{K} z_{k} \alpha_{k} \delta_{k}+e$, where $y$ is the vector of AUC phenotypic observations, $X$ is an incidence matrix of the fixed effects in $\beta$ including group (concatenation of year and sex; four classes), $K$ is the 


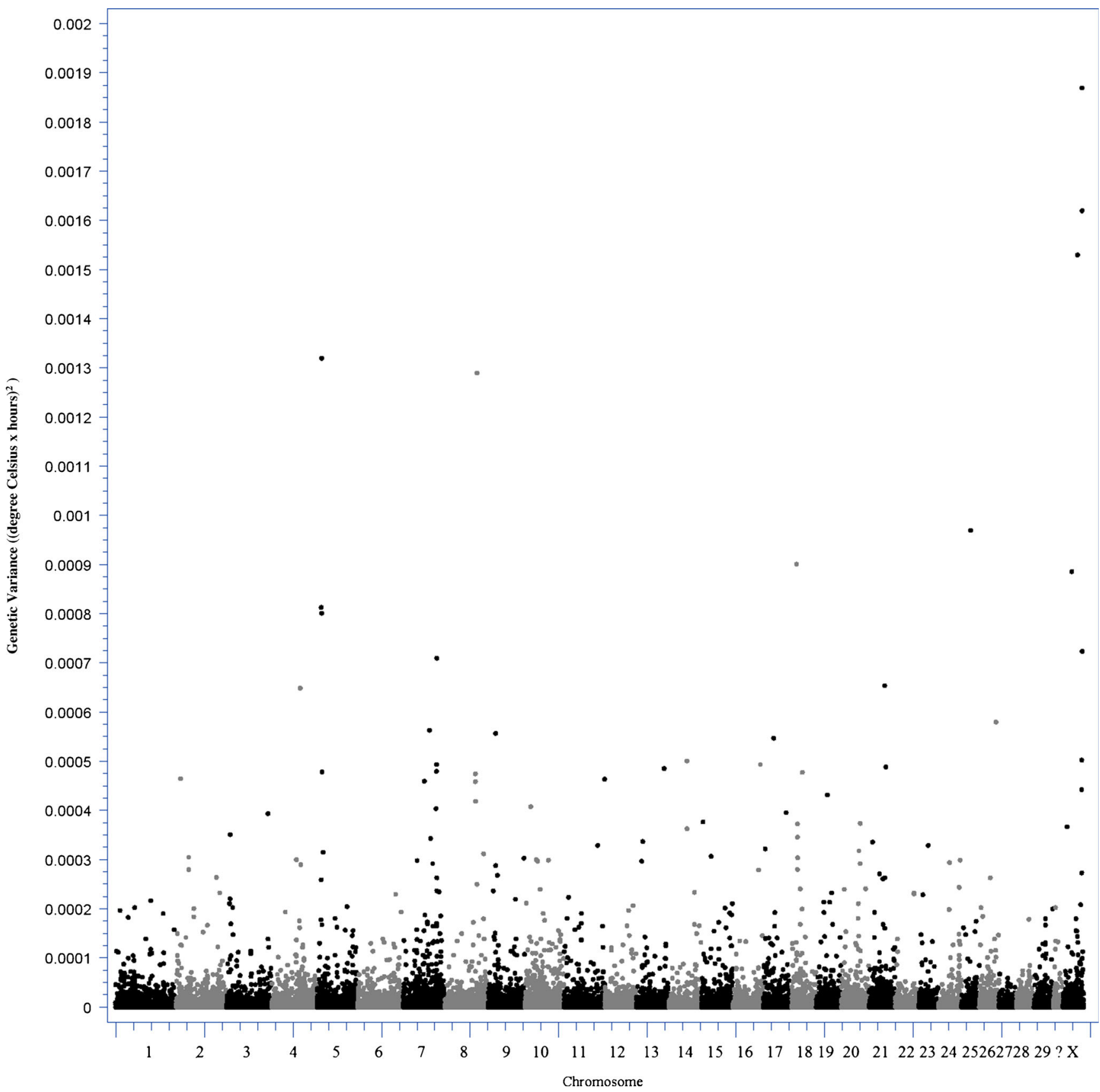

Fig. 2 Genome-wide association analysis between 54,609 SNP and area under the curve across 5 day $(120 \mathrm{~h})$ during winter conditions (AUCW5D). The $Y$-axis represents the contribution of a marker to the

number of markers, $z_{k}$ is the genotype call $(-10,0,10)$ at marker $k, \alpha_{k}$ is the random additive effect of marker $k, \delta_{k}$ is an indicator for whether marker $k$ was included $\left(\delta_{k}=1\right)$ or excluded $\left(\delta_{k}=0\right)$ in the model for a specific iteration of the Markov chain Monte Carlo (MCMC) algorithm, and $e$ is the random residual. The model to estimate marker effects was implemented using BayesC as outlined in Habier et al. (2011). The proportion of markers having a null effect $(\pi)$ was set to 0.995 with prior degrees of
SNP variance ((in degree Celsius $\times$ hours $\left.)^{2}\right)$. On the $X$-axis, alternate gray and black colors represent different chromosomes (chromosome refers BTA1 to BTA29, followed by SNP with unknown location (?)

freedom for the residual and marker variance of 10 and 4, respectively. A chain length of 150,000 iterations was run with the first 50,000 discarded as burn-in. Group was included as a fixed effect in the GWAS to allow for adjustment of different environmental temperatures and temperature collection methods across groups. Although monomorphic SNP were used in the analysis, all monomorphic SNP had a post burn-in genetic variance of zero. Utilizing analysis of variance procedures, group 
accounted for 11.6 and $42.3 \%$ of the total phenotypic variance for AUCS5D and AUCW5D, respectively. The direct genomic value (DGV) of the ith animal was calculated as: $\mathrm{DGV}_{i}=\sum_{k=1}^{K} z_{i k} \widehat{a}_{k}$, where $z_{\mathrm{ik}}$ is the genotype call $\left(-10,0\right.$, and 10) for animal $i$ at marker $k$ and $\widehat{a}_{k}$ is the posterior mean effect at marker $k$.

Convergence was met for all analyses by starting with high and low a priori heritability estimates until the posterior heritability estimates were trending down and up, respectively. When the posterior heritability estimates were trending towards each other a value in the middle was chosen as the a priori heritability. The a priori heritability estimates used for final analyses were 0.68 and 0.2 for AUCS5D and AUCW5D, respectively. The percent of genetic variance explained by a window(s) out of the total genetic variance was calculated by removing all SNP within the window(s) and rerunning the analysis (subset), with the same parameters as the initial run (full). The percentage of variance explained was calculated as 1 - (subset posterior genetic variance/full posterior genetic variance).

Phenotypic correlations between AUCS5D and AUCW5D were estimated using multivariate analysis of variance procedures with group fitted as a fixed effect. To estimate potential re-ranking of animal fitness across temperature stress events, correlations between AUCS5D and AUCW5D DGV were estimated. Additionally, SNP were blocked into $1 \mathrm{Mb}(n=$ 2,677 ) windows and SNP variance within a window was summed. The top $5 \%$ windows $(n=134)$ within each trait were then compared in a similar fashion as the phenotypic and DGV correlation to determine the percentage of windows in common.

\section{Gene ontology}

The position of the SNP with the largest genetic variance from the top $0.5 \% 1-\mathrm{Mb}$ windows $(n=13)$ for each trait were extended by $0.5 \mathrm{Mb}$ in both directions and human orthologs of beef cattle positional candidate genes using Bos taurus assembly (UMD3.1; Ensembl 73) were utilized for functional characterization of individual genes as well as to identify overrepresented gene ontology terms and pathway analysis using DAVID (http://david.abcc.ncifcrf.gov).

Mutations that impacted the functionality of the expressed product of positional candidate genes identified by GWAS and gene ontology analysis were revealed utilizing lowcoverage $(0.5 \times)$ genomic sequences on 96 sires in the USMARC Germplasm Evaluation project cycle VII (GPEVII; Wheeler et al. 2005). The bulls included $49 \mathrm{~F}_{1}$ sires with the most GPEVII progeny and $47 \mathrm{AI}$ sires of $\mathrm{F}_{1}$ bulls. The breeds contained within the sequence data included Angus $(n=13)$, Charolais $(n=11)$, Gelbvieh $(n=16)$,
Hereford ( $n=14)$, Limousin $(n=14)$, Red Angus $(n=14)$, and Simmental $(n=14)$. Heavily used AI sires within the beef industry were utilized in GPEVII, which allowed for the identification of mutations that may be segregating throughout the US beef cattle population. Mutations were classified according to the expected effect on gene function using SnpEff software (Cingolani et al. 2012) with Ensembl (version 68) annotation of the B. taurus assembly (UMD3.1). Criteria for classifying mutations are described in detail on Table 2.

\section{Results}

Figures 1 and 2 display the contribution of each marker to the total SNP variance for AUCS5D and AUCW5D, respectively. The posterior mean heritability $( \pm \mathrm{SD})$ estimated or percentage of variation accounted for by marker genotypes for AUCS5D was $0.68( \pm 0.11)$ and for AUCW5D was $0.21( \pm 0.09)$. Phenotypic and DGV correlations between AUCS5D and AUCW5D were $0.16(P<0.02)$ and $0.40(P<0.001)$, respectively. Standard errors were 0.069 for both the phenotypic and genetic correlation. Despite the moderately positive DGV correlation, only $9.0 \%$ of the top $5 \% 1-\mathrm{Mb}$ windows were

Table 1 Chromosome and position of the top $0.5 \% 1-\mathrm{Mb}$ windows for each trait

\begin{tabular}{lll}
\hline \multirow{2}{*}{ Chromosome } & Trait & \\
\cline { 2 - 3 } & AUCS5D & AUCW5D \\
\hline 1 & $90-91$ & \\
5 & & $8-9,9-10,10-11$ \\
7 & & $88-89$ \\
8 & $43-44$ & $82-83,86-87$ \\
9 & & $22-23$ \\
10 & $91-92$ & \\
11 & $80-81$ & \\
12 & $23-24,25-26,30-31$ & \\
18 & & \\
20 & $17-18$ & $42-43$ \\
21 & & \\
22 & $10-11$ & \\
23 & $50-51$ & \\
25 & $4-5$ & $65-66,117-118,142-143$ \\
26 & $49-50$ & \\
27 & $12-13$ & \\
$X$ & & \\
\hline
\end{tabular}

Position refers to location in megabases $(\mathrm{Mb})$ for a particular chromosome derived from the B. taurus build UMD 3.1 assembly (Zimin et al. 2009). Trait refers to a specific area under the curve (AUC) season where AUCS5D $=$ AUC across 5 day during summer conditions; AUCW5D $=$ AUC across 5 day during winter conditions 
Table 2 Predicted functional effect of the sequence variants derived from low-coverage sequence data within candidate genes located in the extended top $0.5 \% 1-\mathrm{Mb}$ windows

\begin{tabular}{|c|c|c|c|c|c|c|c|c|}
\hline \multirow[t]{2}{*}{ Ensembl gene ID } & \multirow[t]{2}{*}{ Gene } & \multirow[t]{2}{*}{ Function } & \multirow[t]{2}{*}{ BTA } & \multirow[t]{2}{*}{ Position $^{\mathrm{a}}$} & \multirow[t]{2}{*}{ Trait $^{\mathrm{b}}$} & \multicolumn{3}{|c|}{ Magnitude of effect ${ }^{\mathrm{c}}$} \\
\hline & & & & & & 3 & 2 & 1 \\
\hline ENSBTAG00000001605 & $\mathrm{DIO} 2$ & Thyroid hormone regulation & 10 & 92.6 & AUCS5D & 0 & 0 & 0 \\
\hline ENSBTAG00000022991 & NBEA & Body weight and feed intake & 12 & 25.8 & AUCS5D & 2 & 3 & 10 \\
\hline ENSBTAG00000005012 & HSPH1 & Heat shock protein response & 12 & 29.8 & AUCS5D & 0 & 1 & 0 \\
\hline ENSBTAG00000018103 & HMGB1 & Apoptosis & 12 & 30.4 & AUCS5D & 0 & 0 & 0 \\
\hline ENSBTAG00000016566 & ITGA9 & Respiration & 22 & 11.0 & AUCS5D & 1 & 0 & 1 \\
\hline ENSBTAG00000006378 & RIPK1 & Apoptosis & 23 & 50.4 & AUCS5D & 0 & 0 & 3 \\
\hline ENSBTAG00000020109 & TRAP1 & Heat shock protein response & 25 & 2.9 & AUCS5D & 0 & 0 & 0 \\
\hline ENSBTAG00000039555 & $\mathrm{COX} 7 \mathrm{C}$ & Energy metabolism & 7 & 88.6 & AUCW5D & 0 & 0 & 1 \\
\hline ENSBTAG00000009565 & RASA1 & Vasculogenesis & 7 & 89.3 & AUCW5D & 0 & 0 & 0 \\
\hline ENSBTAG00000019554 & FBP2 & Pentose phosphate pathway & 8 & 82.4 & AUCW5D & 0 & 0 & 5 \\
\hline ENSBTAG00000009733 & FBP1 & Pentose phosphate pathway & 8 & 82.5 & AUCW5D & 0 & 0 & 1 \\
\hline ENSBTAG00000024889 & HSBP1 & Heat shock protein response & 18 & 10.1 & AUCW5D & 0 & 0 & 0 \\
\hline ENSBTAG00000020921 & PRKCB & Ion regulation & 25 & 21.8 & AUCW5D & 0 & 0 & 10 \\
\hline ENSBTAG00000001404 & CACNG3 & Ion regulation & 25 & 22.2 & AUCW5D & 0 & 0 & 1 \\
\hline ENSBTAG00000046132 & TRIP11 & Thyroid hormone regulation & 25 & 22.9 & AUCW5D & 5 & 6 & 2 \\
\hline
\end{tabular}

${ }^{\text {a }}$ Position refers to the start position of a gene in megabases for a particular chromosome derived from the B. taurus UMD_3.1 assembly (Zimin et al. 2009)

${ }^{\mathrm{b}}$ Trait refers to a specific area under the curve (AUC) where AUCS5D=AUC across 5 day during summer conditions; AUCW5D=AUC across 5 day during winter conditions

${ }^{\mathrm{c}}$ Magnitude of effect was determined using SnpEff (Cingolani et al. 2012) and 3 (high) refers to a variant resulting in a new start or stop codon, hits a splice donor or acceptor site disrupted a splice site, 1 or many codons are inserted or deleted or an insertion or an insertion or deletion causes a frame shift; 2 (moderate) if a variant resulted in a nonsynonymous amino acid change; 1 (low) if a variant resulted in a synonymous amino acid change

in common between AUCS5D and AUCW5D. The genetic variance explained by $1-\mathrm{Mb}$ windows, based on the posterior marker specific SNP variance, uncovered major regions associated with heat and cold susceptibility. The chromosomes and position of the top $0.5 \% 1-\mathrm{Mb}$ windows for AUCS5D and AUCW5D are outlined in Table 1. The top $0.5 \% 1-\mathrm{Mb}$ windows $(n=13)$ explained 7.1 and $6.8 \%$ of the genetic variance for AUCS5D and AUCW5D, respectively.

Functional annotation, enrichment, and pathway analysis of the extended regions of the top $0.5 \% 1-\mathrm{Mb}$ windows that harbored SNP associated with the largest effect resulted in enrichments for cardiac muscle contraction $(P<0.05 ; C O X 7 C$, COX4I1, and $C A C N G 3)$ and pentose phosphate $(P<0.07$; $F B P 1$ and $F B P 2$ ) pathways for AUCW5D. Enrichment for AUCS5D was detected in gap junction $(P<0.12 ; T U B B 2 A$ and $T U B B 2 B)$. Individual candidates in these regions included genes involved in cellular response to stress $(C C N H$ and TNRC6A), apoptosis (FGD3, G2E3, RASA1, CSTB, and $D A P K 1)$, ion transport (CACNG3,CLCN4,PRKCB, and TRPC5), and generation of precursor metabolites and energy (COX4II and $C O X 7 C$ ) for AUC5WD and cellular response to stress (STAC, WRNIP1, MLH1, RIPK1, SMC6, and GEN1), response to heat (STAC), apoptosis (MLH1, RIPK1, and SERPINB9), and ion transport (KCNS3, SLC22A23, and TRPC4) for AUCS5D. High, moderate, and low impact variants within the extended top $0.5 \% 1-\mathrm{Mb}$ windows were revealed via low-coverage sequence data within candidate genes located via DAVID or genes with functions related to heat or cold stress are outlined in Table 2.

\section{Discussion}

The posterior mean heritability for AUCS5D was larger than previous estimates of 0.11 to 0.44 , which used one-time measurements of rectal temperature as an indicator of heat stress (Burrow 2001; Da Silva 1973; Turner 1982, 1984). The high posterior heritability estimates may be attributed to associations between markers and AUC phenotypes occurring due to using an admixed population or attributed to the small sample size. The slightly positive phenotypic correlation indicates heat tolerant animals are more likely to be cold intolerant, or vice-versa. Furthermore, the moderately positive DGV correlation indicates that selection for heat tolerance (low AUC value) may result in animals that are cold intolerant 
(low AUC value), or vice versa. A limited number of the top $5 \% 1-\mathrm{Mb}$ windows were in common across season $(9.0 \%)$, suggesting that with the aid of genomics, these antagonisms can be broken.

Functional annotation, enrichment, and pathway analysis uncovered regions that involved genes underlying how cells respond to cellular stress, heat stress, either through protective roles of pentose phosphate pathway, heat shock proteins, or apoptosis. Pentose phosphate pathway generates NADPH used to replenish oxidized glutathione, which is necessary to avoid the damaging effects of reactive oxygen species on macromolecules under stress conditions (Go and Jones 2008). The expression of two members of the pentose-phosphate pathway was impacted by heat stress in a study of the response to acute heat stress of two species of mussels that displayed differences in heat sensitivity (Tomanek and Zuzow 2010). Also, during systematic and/or cellular stress, heat shock proteins function as molecular chaperones, which enhance the protein folding capacity of a cell, thus counteracting the stress and promoting cell survival (Fulda et al. 2010). In a study by Ju Oh et al. (1997), it was shown that in vivo overexpression of heat shock 105/110 kDa Protein 1 (HSPH1) conferred substantial heat resistance to both Rat-1 and HeLa culture cell lines. Also, regions associated with body weight (NBEA; Olszewski et al. 2012), respiration (ITGA9; Chen et al. 2012), and thyroid function (DIO2) were found for AUCS5D. Furthermore, functional annotation of the major 1-Mb windows uncovered genes involved in ion transport for both AUCS5D (KCNS3, SLC22A23, and TRPC4) and AUCW5D (CACNG3, CLCN4, PRKCB, and TRPC5). The genes involved in ion transport involved the directed movement of $\mathrm{Ca}^{2}, \mathrm{Na}^{+}$, and $\mathrm{K}^{+}$, all of which have important functions in increasing heat production via ion leaks (Himms-Hagen 1976). The peak associated with the X-chromosome in Figs. 1 and 2 was further investigated by carrying out a GWAS within each sex and the peak was still observed, which indicated the peak was not due to an unaccounted for sex effect. Overall, the sequence information revealed multiple high to low impact mutations within candidate genes. The mutations had functions related to pentose phosphate pathway ( $F B P 1$ and $F B P 2$ ), ion regulation ( $P R K C B$ and $C A C N G 3)$, body weight and feed intake (NBEA), respiration (ITGA9), apoptosis (RIPK1), heat shock protein response ( $H S P H 1)$, and generation of precursor metabolites and energy $(C O X 7 C)$. These results provide a broader base of potential genes and pathways along with the moderate to high impact mutations within these genes to further investigate their possible roles in heat and cold stress in other populations. Differences in genetic susceptibility to environmental stress have been seen in comparative studies between Bos indicus and B. taurus breeds (Hansen 2004; Kamwanja et al. 1994), but a limited amount of research has been done on identifying genes or genetic pathways within a population on a genome-wide level.

\section{Conclusion}

Medium-density genomic information was able to describe a moderate to large proportion of the phenotypic variation in BT for the current population during periods of heat and cold stress. There was a moderate positive DGV correlation between heat and cold stress, with relatively few genomic regions that had an effect on both heat and cold stress. Genomic regions affecting $\mathrm{BT}$ regulation during heat and cold stress have been identified and have been found to harbor multiple candidate genes with high- to low-impact mutations, although further evaluation of these regions is needed in other populations.

Acknowledgments The authors would like to thank Leslie J. Johnson, Stephanie Moore, Cody Schneider, and Brandon Nuttelman for their assistance during data collection and the University of NebraskaLincoln Layman award for funding of the project.

\section{References}

Bergen RD, Kennedy AD (2000) Relationship between vaginal and tympanic membrane temperature in beef heifers. Can J Anim Sci 80:515-518

Burrow HM (2001) Variances and covariances between productive and adaptive traits and temperament in a composite breed of tropical beef cattle. Livest Prod Sci 70:213-233

Chen C, Kudo M, Rutaganira F, Takano H, Lee C, Atakilit A, Robinett KS, Uede T, Wolters PJ, Shokat KM, Huang X, Sheppard D (2012) Integrin $\alpha 9 \beta 1$ in airway smooth muscle suppresses exaggerated airway narrowing. J Clin Invest 122(8):2916-2927

Cingolani P, Platts A, Wang LL, Coon M, Nguyen T, Wang L, Land SJ, Lu X, Ruden DM (2012) A program for annotating and predicting the effects of single nucleotide polymorphisms, SnpEff: SNPs in the genome of Drosophila melanogaster strain w1118; iso-2; iso-3. Fly 6:80-92

Da Silva RG (1973) Improving tropical beef cattle by simultaneous selection for weight and heat tolerance. J Agric Sci 96:23-28

Edriss V, Guldbrandtsen B, Lund MS, Su G (2012) Effect of marker-data editing on the accuracy of genomic prediction. J Anim Breed Genet 130:128-135

Fernando RL, Garrick DJ (2009) GenSel user manual for a portfolio of genomic selection related analysis. Animal breeding and genetics. Iowa State University, Ames

Fulda S, Gorman AM, Hori O, Samali A (2010) Cellular stress responses: cell survival and cell death. Int J Cell Biol 2010:1-23

Go YM, Jones DP (2008) Redox compartmentalization in eukaryotic cells. Biochim Biophys Acta 1780:1273-1290

Habier D, Fernando RL, Kizilkaya K, Garrick DJ (2011) Extension of the Bayesian alphabet for genomic selection. BMC Bioinforma 12:186

Hahn GL (1999) Dynamic responses of cattle to thermal heat loads. J Anim Sci 77:10-20

Hansen PJ (2004) Physiological and cellular adaptations of zebu cattle to thermal stress. Anim Reprod Sci 82-83:349-360

Himms-Hagen J (1976) Cellular thermogenesis. Ann Rev Physiol 38: 315-351

Howard JT, Kachman SD, Nielsen MK, Mader TL, Spangler ML (2013) The effect of myostatin genotype on body temperature during extreme temperature events. J Anim Sci 91:3051-3058 
Illumina, Inc. (2010) Technical note: DNA analysis, Infinium ${ }^{\circledR}$ genotyping data analysis. San Diego (CA). Illumina, Inc. 9 p. pub. no. 9702007-005

Ju Oh H, Chen X, Subjeck JR (1997) Hsp110 protects heat-denatured proteins and confers cellular thermoresistance. J Biol Chem 272: 31636-31640

Kamwanja LA, Chase CC Jr, Gutierrez JA, Guerriero V Jr, Olsen TA, Hammond AC, Hansen PJ (1994) Response of bovine lymphocytes to heat shock as modified by breed and antioxidant status. J Anim Sci 72:438-444

Mader TL, Johnson LJ, Gaughan JB (2010) A comprehensive index for assessing environmental stress in animals. J Anim Sci 88:2153-2165

MacNeil MD (2009) Invited review: research contributions from seventyfive years of breeding line 1 Hereford cattle at miles city, Montana. J Anim Sci 87:2489-2501

Nardone A, Valentini A (2000) The genetic improvement of dairy cows in warm climates. Proceedings of the joint ANPAEAAP-CIHEAM-FAO symposium on Livestock production and climatic uncertainty in the Mediterranean. Agadir, Morocco. EAAP Publication no. 94, 2000

Olszewski PK, Rozman J, Jacobsson JA, Rathkolb B, Strömberg S, Hans W, Klockars A, Alsiö J, Risèrus U, Becker L, Hölter SM, Elvert R, Ehrhardt N, Gailus-Durner V, Fuchs H, Fredriksson R, Wolf E, Klopstock T, Wurst W, Levine AS, Marcus C, Hrabě de Angelis M, Klingenspor M, Schiöth HB, Kilimann MW (2012) Neurobeachin, a regulator of synaptic protein targeting, is associated with body fat mass and feeding behavior in mice and body-mass index in humans. PLoS Genet 8(3):e1002568. doi:10.1371/journal. pgen. 1002568

Scharf B, Carroll JA, Riley DG, Chase CC, Coleman SW, Keisler DH, Weaber RL, Spiers DE (2010) Evaluation of physiological and blood serum differences in heat-tolerant (Romosinuano) and heatsusceptible (Angus) Bos taurus cattle during controlled heat challenge. J Anim Sci 88:2321-2336

Tomanek L, Zuzow MJ (2010) The proteomic response of the mussel congeners Mytilus galloprovincialis and $M$. trossulus to acute heat stress: implications for thermal tolerance limits and metabolic costs of thermal stress. J Exp Biol 15:3559-3574. doi:10.1242/jeb. 041228

Turner HG (1982) Genetic variation of rectal temperature in cows and its relationship to fertility. Anim Prod 35:401-412

Turner HG (1984) Variation in rectal temperature of cattle in a tropical environment and its relation to growth rate. Anim Prod 38:417-427

Wheeler TL, Cundiff LV, Shackelford SD, Koohmaraie M (2005) Characterization of biological types of cattle (cycle VII): carcass, yield, and longissimus palatability traits. J Anim Sci 83:196-207

Young BA (1983) Cold stress as it affects animal production. J Anim Sci 52:154-163

Zimin AV, Delcher AL, Florea L, Kelley DR, Schatz MC, Puiu D, Hanrahan F, Pertea G, van Tassell CP, Sonstegard TS, Marcais G, Roberts M, Subramanian P, Yorke JA, Salzberg SL (2009) A wholegenome assembly of the domestic cow, Bos taurus. Genome Biol 10(4):R42 